\title{
Studies on the site of electron transfer to triphenyl- tetrazolium chloride in rat liver mitochondria with reappraisal of Nachlas-Seligman's theory
}

\author{
Toshifumi OTORI \\ Department of Ophthalmology, Osaka University Medical School
}

A study was made of the site of electron transfer to triphenyltetrazolium chloride (TTC) in mitochondrial electron transport system, with critical evaluation of Nachlas-Seligman's theory that TTC is reduced at the cytochrome oxidase level. Indirect spectrophotometric assay of TTC-formazan and polarographic determination of oxygen concentration of the medium by the use of a Hagihara's oxygen electrode revealed the following results :

1) Endogenous TTC-reductase activity was not detected when the reaction was carried out using an open vessel, but it was observed definitely when a Thunberg tube was evacuated and flushed with nitrogen gas. This endogenous activity which was detectable in the assay using the Thunberg tube was not inhibited by $\mathrm{KCN}$, Antimycin A and malonate.

2) Succinate TTC-reductase activity was not increased by evacuation of the tube and/or flushings with nitrogen gas. Total TTC-redutase activity was increasd, but this increase corresponded to an increase in endogenous TTCreductase activity of mitochondria in anaerobiosis.

3) There was observed a time lag prior to the appearance of succinate TTC-reductase activity of mitochondria in the case of open vessel reaction. This time lag seemed to correspond to the time required for mitochondria to consume the total oxygen in the solution.

4) Succinate TTC-reductase was almost completely inhibited by $\mathrm{KCN}$ and Antimycin A, but this inhibition was markedly removed by evacuation of the tube. Carbon monoxide produced a pronounced inhibition of succinate TTCreductase when the reaction was carried out in the tube flushed with $1096 \mathrm{O}_{2}$ $90 \% \mathrm{CO}$ gas, while this inhibition was also removed by evacuation and flushings with $100 \%$ CO gas.

5) Malonate inhibition of TTC-reductase was significantly decreased by evacuation and flushing with nitrogen gas.

6) TTC did not decrease, or rather increased oxygen uptake of mitochondria. It was found that an addition of TTC or freezing-thawing of mitochondria which had showed respiratory control uncoupled their oxidative phosphorylation.

7) TTC was not reduced by mitochondria with p-phenylene diamine or hydroquinone as substrates.

8) Purified cytochrome oxidase preparation (cytochrome c+ cytochrome a), first prepared by Prof. Okunuki et al, did not reduce TTC at all with hydroquinone as substrate, although it showed an intense oxygen uptake. 
Except for the above investigations on liver mitochondria, purified xanthine oxidase and yeast lactic dehydrogenase were studied as to their reducibility of TTC and INT using direct spectrophotometric assay method. It was revealed that both of the enzymes began to reduce TTC when oxygen concentration of the solution was decreased to $0 \%$, but that they started reducing INT with no relation to oxygen concentration of the solution.

It was thus concluded that TTC was reduced by mitochondria at the flavoprotein level after the oxygen concentration of the solution was decreased, even in the case of open vessel reaction, to $0, \%$ by the oxygen uptake of mitochondria, and that cyanide and Antimycin A inhibition of succinate TTCreductase was a spurious or pretended inhibition at the cytochrome oxidase level.

It was also suggested that TTC and INT were reduced by not only flavoprotein of mitochondria but also xanthine oxidase and yeast lactic dehydrogenase in accordance with the reduction oxidation potential theory.

\title{
Discussion
}

Dr. Ogawa :

1. Did you study with any other tetrazolium salts than TTC ?

2. Of Nachlas's opinion, it is the essentials that different kinds of tetrazolium salts accept electron through different level of electron transfer system. Dr. Otori did not discuss about this problem.

Dr. Otori :

The present study has shown that TTC is not reduced at the cytochrome oxidase level. Other tetrazoles will be examined in future.

Dr. Mizutani :

1) Did you get any findings with the addition of amytal into the reaction mixtures ?

2) About lactic dehydrogenase, we must be cautious to the different biological characters between yeast and animals, i. e. DPN-independent and dependent.

Dr. Otori :

I have not examined the action of amytal.

\section{Studies on the metachromasia with cyanine dyes}

\author{
Akira OKADA \\ The Department of Pathology, Tuberculosis Research \\ Institute, Kyoto University, Kyoto, \\ (chief : Prof. H. Takamatsu)
}

On the study of the so-called metachromaisa most work has been actually done with thiazine dyes especially toluidine blue or azure A. However, it has been known that many other dyes give metachromatic effect on the specialized chromotropes. 